\title{
CUADRO DE MANDO PARA UN DESTINO TURÍSTICO INTELIGENTE
}

\author{
Díaz S. \\ Dpto. de Ingeniería Informática y de Sistemas. Universidad de La Laguna, sdiazgon@ull.edu.es \\ Torres, J.M. \\ Dpto. de Ingeniería Informática y de Sistemas. Universidad de La Laguna, jmtorres@ull.edu.es \\ Aguilar, R.M. \\ Dpto. de Ingeniería Informática y de Sistemas. Universidad de La Laguna, raguilar@ull.edu.es \\ Parra, E. \\ Dpto. de Economía y Dirección de Empresas. Universidad de La Laguna, eparra@ull.edu.es
}

\begin{abstract}
Resumen
Mejorar siempre es una ardua tarea que precisa de nuevas herramientas y/o técnicas, que permitan al individuo o grupo de estos poder dar una vuelta de tuerca a determinada situación y obtener una nueva visión de los problemas a enfrentar. En concreto, en actividades relacionadas al sector económico, como lo es el sector turístico, la extrema competencia conlleva no sólo la necesidad de mejorar cada día, sino la obligación de ello. Por esto, predecir el rumbo del mercado y tomar las decisiones acertadas en cada momento y coyuntura es imperiosamente necesario. Procesos que van estrechamente ligados con la información con la que trabajen los decisores. En la actualidad estamos viviendo una explosión en cuanto a número de fuentes de información. Pero ello, además de una ventaja, trae consigo un problema: se encuentran diversificadas en diferentes lugares y formatos. En este trabajo se presenta un cuadro de mando que permite la visualización datos reunidos, de forma automática, provenientes tanto de fuentes estructuradas y no estructuradas del sector turístico en Tenerife (Islas Canarias), analizados y clasificados. Todo conformado por herramientas de software libre.
\end{abstract}

Palabras clave: cuadro de mando, redes sociales, proceso analítico jerárquico, indicador clave de rendimiento turismo

\section{INTRODUCCIÓN}

El sector turístico es un sector en continua evolución, donde es necesaria realizar una tarea de predicción, ya que esta industria es muy susceptible a cambios. Que en un año obtengan excelentes resultados no implica que en los venideros se vaya a repetir dicho éxito aunque se utilicen las mismas estrategias. Es por ello que la toma de decisiones informada, con toda la información necesaria para realizar un buen análisis, resulta un gran reto. En la actualidad, existe una gran cantidad de datos e informes provenientes de múltiples fuentes de información, siendo muy laboriosa la tarea de ir recopilando todo estos datos oficiales sobre los destinos turísticos. Además hoy en día se cuenta con nuevas fuentes de datos, no menos importantes que las oficiales: las redes sociales. Y es que hablamos de información directa del turista de la que se puede extraer conclusiones muy valiosas. Por tanto el problema de la extracción, tratamiento y carga (ETL) de estos datos se antoja esencial en la predicción del comportamiento futuro del mercado. Y resulta extremadamente peligroso, competir en mundo tan exigente sin tener todos los datos necesario para la toma de decisiones correcta. Cada destino compite ferozmente con los demás. Cada uno centrándose en una serie de estrategias a desarrollar para captar turistas. Unos promocionándose como destino de sol y playa, otros de bienes histórico-culturales, otros promocionando sus bienes naturales; unos intentando captar al mayor número de turistas posible, otros centrándose en atraer a turistas de alto standing, etc. Cada estrategia puede ser válida, dependiendo de los criterios de cada destino, pero la pregunta a hacerse es, ¿cómo saberlo? ¿Cómo tener la certeza de que las estrategias elegidas van ser exitosas? ¿Cómo medir el éxito de un conjunto estrategias? Y si crees que no se están obteniendo los resultados, ¿cómo averiguar dónde se está fallando?

Tenerife hoy en día es un destino que atrae a más de 5 millones de turistas al año. La mayoría de turistas se hospedan en la zona sur de la isla y aunque el recurso que recurrentemente se ha promocionado a lo largo de los años ha sido el favorable 
clima con el que cuentan las islas, no hay que menospreciar al Parque Nacional del Teide, Patrimonio de la Humanidad, el más visitado de España y uno de los más visitados del mundo. Además de los parques rurales de Anaga y de Teno y los diferentes cascos históricos y parques temáticos. Hoy en día la recuperación en el sector del turismo por parte de los países norteafricanos y Turquía, además del despegue de nuevos competidores como Marruecos, amenaza la época de bonanza turística que se ha vivido en estos últimos años en las islas. Esto conlleva la búsqueda de nuevas estrategias y por tanto nuevas formas de optimizar la toma de decisiones. Y es aquí donde entran las nuevas tecnologías.

El modus operandis hoy en día ante la toma de decisiones consiste en analizar los diferentes informes que se reciben de entidades públicas, como son los informes del Instituto Nacional de Estadística o los que redactan las consejerías de turismos de las diferentes Comunidades Autónomas y otros organismos, además de los privados que pueda realizar el propio interesado. Informes provenientes de diversos organismos pero que analizan los mismos indicadores y que sin embargo muestran resultados diferentes entre unos y otros. ¿Cuál es el más fiable? Existen entidades españolas que cuentan con aplicaciones capaces de volcar los informes de las diferentes fuentes de información en un único lugar. En concreto, hablamos de la isla de [2] o de la ciudad de [1]. Pero son aplicaciones que sólo agrupan informes venidos de fuentes oficiales con datos estructurados. Pero en este trabajo, proponemos incluir las fuentes de datos no estructurados, principalmente redes sociales, que no se están analizando hoy en día. Con todo lo que conllevaría poder acceder a esa valiosa información, tratada y analizada de forma automática.

Algunas empresas privadas, como pueden ser hoteles o restaurantes, tienen personal, específico o no, encargado de acceder manualmente a Facebook o Tripadvisor (entre otros) y leer una a una las opiniones de los clientes para poder llegar a conclusiones sobre su negocio. Lo que ya de por sí supone un gran gasto de personal y tiempo. Pero trasladado a un escenario más grande, por ejemplo una ciudad o una isla, proceder a intentar realizar el mismo análisis manual con todas opiniones es más que inviable.

Por tanto, si se quiere dar un salto cualitativo, es necesaria una herramienta capaz de poder reunir en un único lugar todo esta Big-Data, analizarla y clasificarla para en última instancia visualizar los resultados en una aplicación. Y así permitir que el grupo gestor pueda tomar las decisiones en las mejores condiciones posibles.
Big-Data es el proceso que conlleva la recopilación de cantidades de datos enormes y el análisis de los mismos con el fin de encontrar información oculta, patrones recurrentes, nuevas correlaciones, etc. Para ello, en este proyecto se desplegó la infraestructura necesaria para almacenar e indexar en una base de datos información automáticamente. Hay múltiples opciones en el mercado, tanto de software libre como de pago, pero se optó por la plataforma Elasticsearch para satisfacer nuestras necesidades. Esto es debido a la escalabilidad que proporcionan sus clusters, que permiten manejar terabytes de datos sin ningún problema. Además se instaló la herramienta Airflow para gestionar el flujo de trabajo, ejecutando automáticamente y en un orden establecido anteriormente la pila de tareas que permiten la automatización del proceso. Por otro lado contamos con Kibana para poder visualizar los datos de forma interna gráficamente, mediante una interfaz simple y óptima. Todos estos servidores incluidos en una infraestructura mediante de contendedores Docker.

En este trabajo en concreto se presenta el modelo a partir del cual se realiza la extracción de los datos, la técnica empleada para clasificar los datos y poder cuantificarlos, y las herramientas utilizadas para crear el cuadro de mando final en donde los expertos del sector van a poder visualizar estos resultados. 


\section{MODELO DE VISUALIZACIÓN: CUADRO DE MANDO}

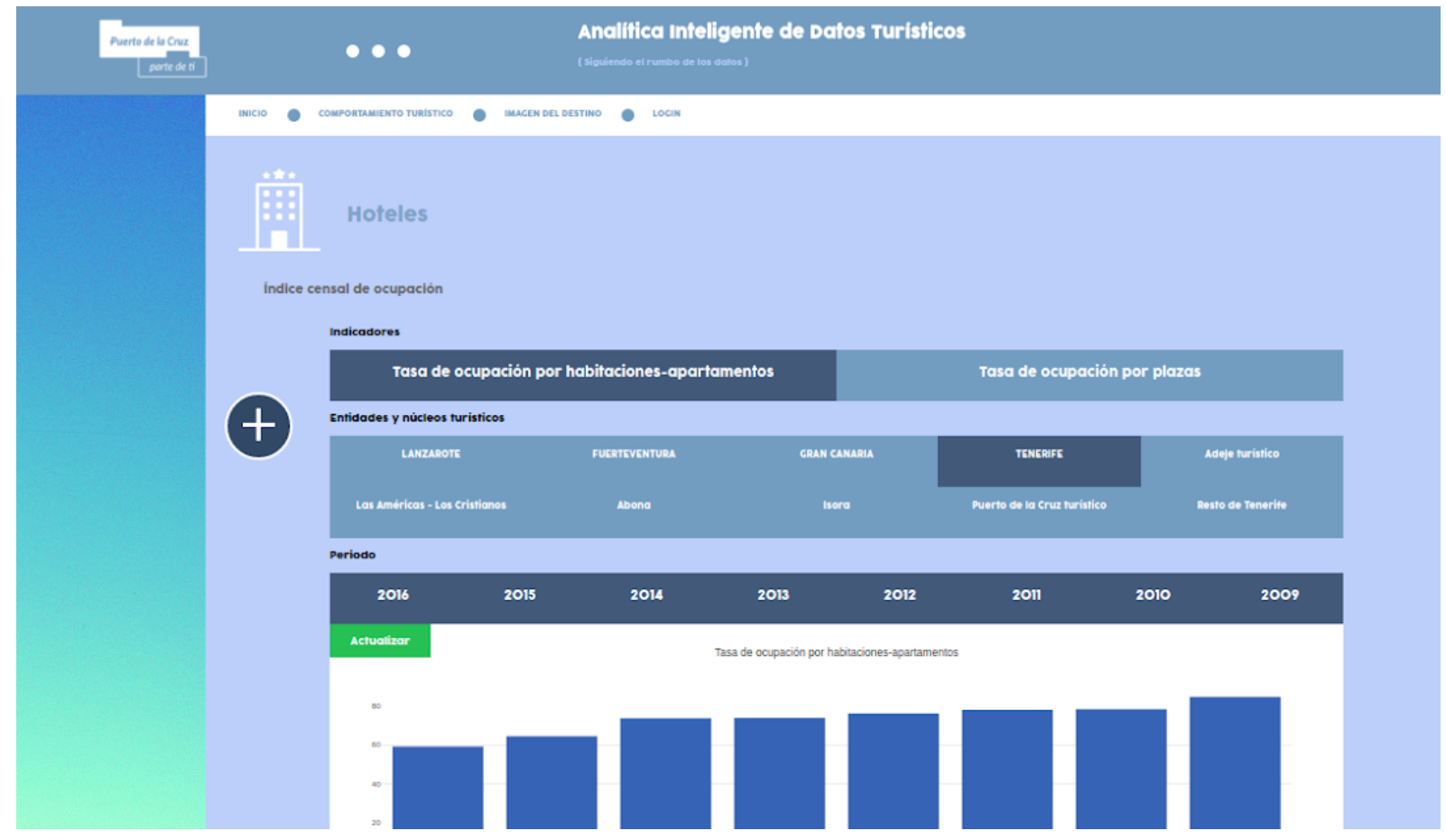

Figura 1: Ejemplo de página del cuadro de mando. En concreto, corresponde con el KPI Hoteles, agrupado dentro de Comportamiento Turístico.

Un cuadro de mando, o cuadro de mando, es una representación gráfica de los principales indicadores o KPIs (key performance indicator), que intervienen en la consecución de los objetivos de un negocio, y están orientados a mejorar la toma de decisiones, minimizando errores. Siendo los KPIs una medida del nivel del rendimiento de un proceso, diseñados para mostrar cómo es el progreso en un proceso o producto en concreto.

Para conseguir un cuadro de mando eficiente es necesario elegir bien los KPIs, que informen de la dinámica del proceso y aporten valor para la consecución de los objetivos planteados, permitiendo tomar las decisiones oportunas en mejor posición. Además, es importante la visualización de las mismos, pues los gestores deben ser capaces de interpretar fácilmente la información que se les está mostrando. Por tanto el cuadro de mando debe de ser breve, con el mismo lenguaje que el decisor y con una representación gráfica adecuada y agradable visualmente, totalmente personalizado. Es una herramienta indispensable para monitorizar las estrategias de la empresa. No sirve directamente para definir la acción correcta concreta a desarrollar pero sí para optimizar la toma de decisiones:
- Permite hacer un seguimiento del grado de cumplimiento de los objetivos del negocio.

- Es la muestra objetiva de los indicadores del negocio.

- Debe guiar e identificar el origen de una campaña positiva o negativa, permitiendo una decisión al respecto, aunque no te va a detallar el porqué de la misma.

En este caso, orientado al turismo en Tenerife, se ha creado dos grandes grupos de KPIs: Comportamiento Turístico e Imagen del Destino. En Comportamiento Turístico se agrupan los KPIs que muestran cómo ha sido la conducta del turista durante sus vacaciones. Por contra, a Imagen del Destino están asociados aquellos KPIs que muestran la percepción de turista e indicadores generales del destino, Tenerife. Cada grupo grupo consta con las siguientes KPIs, que a su vez son nodo padre de otro grupo de KPIs más específicas que los componen, en forma de árbol jerárquico. Todas estas KPIs consensuadas con expertos del sector turístico y son flexibles. Esto significa que están abiertas a los cambios que pueda acontecer el sector, modificándose si es necesario. Comportamiento Turístico cuenta con: 
- Visitantes. Relacionado con el comportamiento del visitante, como por ejemplo la estancia media en el destino, los motivos por el que lo eligen o la cantidad de turistas por país o región de residencia.

- Indicadores de Hoteles. Nos permite conocer como repercute en los complejos hoteleros el turismo en una zona, por ejemplo viendo sus ingresos o la cantidad de plazas que ofertan.

- Indicadores de Gasto y Consumo. Muestra el gasto y consumo, ya sea diario o total, del turista en el destino según diversos factores como edad, procedencia, sexo, etc.

- Indicadores de Restauración. Nos permite saber como es la reputación de los restaurantes del destino.

- Eventos y Museos. Muestra la correlación de los eventos concretos del destino y sus museos en el turismo.

- Indicadores de Movilidad. Indica cómo y por dónde transitan los visitantes del destino.

- Sanidad y OIT. Establece por un lado la correlación entre los turistas y el servicio sanitario y por el otro el uso de las oficinas de información turísticas.

- Indicadores de creación de productos. Enseña los distintos productos turísticos del destino $\mathrm{y}$ actividades a realizar y su relación con el turista.

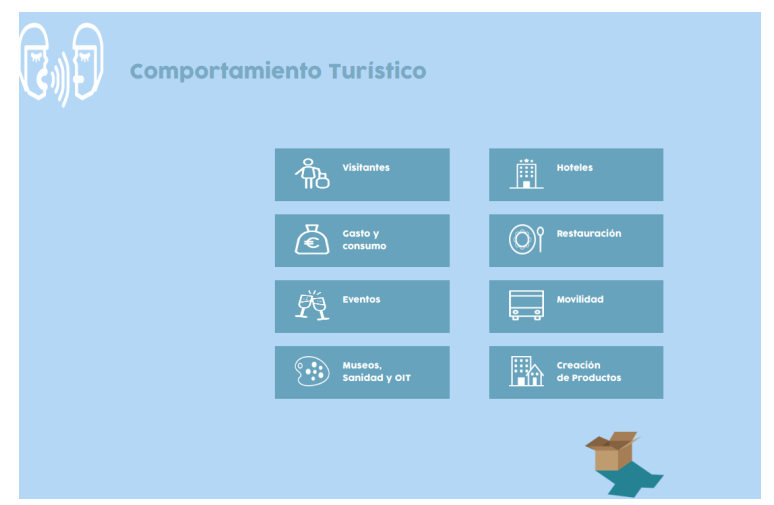

Figura 2: Conjunto de KPIs para Imagen del Destino.

Y por otra parte Imagen del Destino cuenta con:

- Redes Sociales. Muestra indicadores que miden la imagen del destino que tienen los turistas en redes sociales.
- Recursos Naturales. Agrupa los diferente elementos relacionados con la naturaleza del lugar, como pueden ser indicadores del clima, playas, especies locales, etc.

- Infraestructuras Generales. Reúne las diferentes infraestructuras que tiene el destino, como puede ser carreteras, aeropuertos o puertos.

- Infraestructuras Turísticas. Aglutina las infraestructuras turísticas, hoteles, alquiler vacacional, restaurantes, discotecas, etc.

- Factores Económicos. Aquellos factores que caracterizan al destino, empleo, contratos, desarrollo económico o seguridad.

- Entorno Natural. Asocia el destino con sus lugares turísticos, que pueden ser desde monumentos naturales hasta parques de atracciones.

- Entorno Social. Indica el desarrollo de la sociedad local: idiomas, hospitalidad, ingresos, etc.

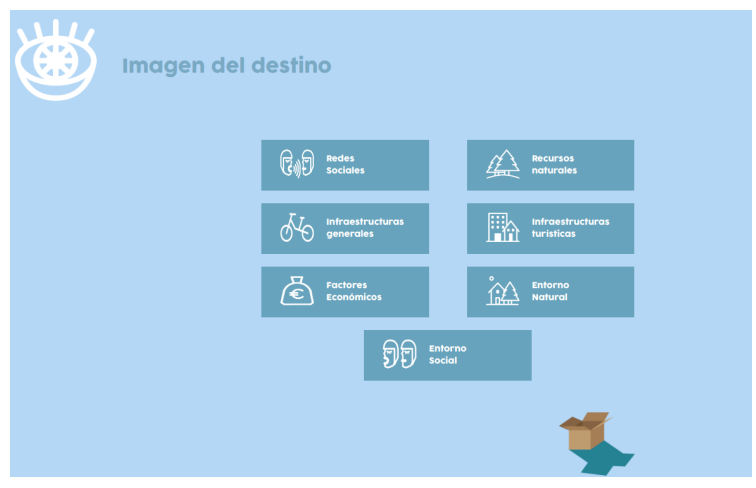

Figura 3: Conjunto de KPIs para Imagen del Destino.

Los datos de cada KPI se obtienen de diversas fuentes que se pueden clasificar según su procedencia en dos tipos: datos estructurados y datos no estructurados.

Los datos estructurados son aquellos que se obtienen a partir de fuentes oficiales con formato definido. Fuentes como son los diferentes institutos de estadísticas, como el INE (Instituto Nacional de Estadística) o el ISTAC (Instituto Canario de Estadística). Y formatos como pueden ser hojas de cálculo o csv (del inglés comma-separated values). Pero para obtener un cuadro de mando totalmente funcional con los datos estructurados no es suficiente. Se está dejando de lado gran cantidad de información útil y fundamental para tomar decisiones. 
Las redes sociales desempeñan un papel muy relevante en el mundo del turismo. Desde las redes sociales convencionales, Facebook, Twitter, Instagram hasta las especializadas como Tripadvisor, Booking, AirBnB, se puede observar la opinión directa del cliente, su comportamiento y la capacidad de las infraestructuras turística entre otras cosas. Es indispensable clasificar toda la información que se pueda obtener de éstas para poder hacer un diagnóstico mejorado del destino, definiendo mucho mejor debilidades y fortalezas que cuando sólo se utilizan fuentes estructuradas. Se puede deducir desde por qué gusta tanto cierta zona de una ciudad, hasta cuánta reputación tiene cierta playa o cuál es el restaurante más popular. Basta con aplicar técnicas de análisis de sentimientos de los posts/tuits/comentarios, obteniendo la cuantía positiva-neutra-negativa del mensaje, además de recoger indicadores númericos, como por ejemplo pueden ser la cantidad de me gustas a un post de Facebook o la cantidad de visualizaciones que ha obtenido.

\section{PRIORIZACIÓN DE LOS INDICADORES (KPI): AHP}

Para hacer más precisa la toma de de decisiones se ha implementado la técnica AHP (Analytic Hierarchy Process) [3]. Una técnica estructurada mul- ticriterio discreta que pretende proporcionar una evaluación cuantitativa para las alternativas inherentes a problemas en los que concurren varios criterios sin necesidad de que los decisores hagan explícitas sus preferencias ni cuantifiquen exhaustivamente la contribución de cada alternativa en cada momento. Las preferencias, o más adecuadamente la prelación de cada alternativa frente al resto, se infieren a partir de comparaciones sucesivas codificadas de acuerdo con una escala.

AHP opera de acuerdo con tres principios:

- Descomposición jerárquica: el problema se estructura a modo de jerarquía, con el objetivo en la cúspide, las alternativas en el escalón inferior, y los criterios en el nivel (o niveles) intermedios.

- Juicio comparativo: todos los elementos de la jerarquía se evalúan por parejas, comparándolos en relación a los elementos del nivel inmediatamente superior de la jerarquía; estas comparaciones se traducen en juicios codificados

- Síntesis: los juicios se agregan en vectores de prioridades dentro de cada nivel, y finalmente se integran para dar lugar a una clasificación general final.

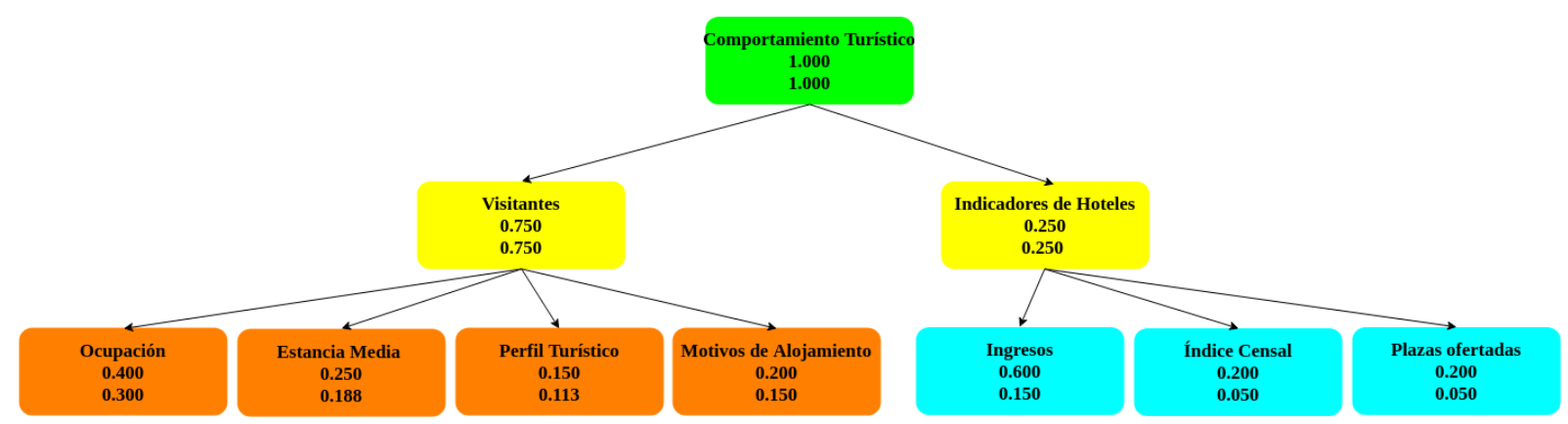

Figura 4: Funcionamiento de la técnica AHP. La suma de las prioridades de los hijos deben dar valor a la unidad. El primer valor de cada recuadro se corresponde a la prioridad de ese itinerario respecto al primer nodo padre. El valor de debajo es la prioridad respecto al nodo padre principal, es decir, Comportamiento Turísitico en este caso.

En definitiva el AHP es beneficioso para las siguientes situaciones:

- Selección. Te ayuda a escoger entre distintas alternativas dadas donde existen múltiples criterios en la decisión.

- Clasificación. Ordenador un conjunto de alternativas desde el más deseable hasta el que menos.

- Priorización. Determinar prioridades entre un conjunto de alternativas en vez de seleccionar sólo una o clasificarlas.

- Asignación de recursos. Posibilitar la repartición de recursos entre distintas alternativas.

- Benchmarking. Comparar los procesos/tareas de una organización con otra para determinar en cuál es mejor.

- Gestión de calidad. Manejar aspectos multidimensionales de la calidad y la mejora de la 
misma.

Una jerarquía es un sistema de clasificación y organización de personas, tareas, ideas, pautas, etc., donde cada uno de los elementos del sistema, exceptuando el primero de la pirámide, es subordinado por uno o más elementos. En el caso que nos ocupa, la valoración del destino encabeza la jerarquía, para luego dividirse entre los dos grupos de KPIs, Comportamiento Turístico e Imagen del Destino y estas a sus vez en sus diferentes items.

Una vez tenemos la jerarquía, los decisores evalúan sus elementos para compararlos unos con otros, pero únicamente se comparan dos elementos a la vez. Para estas comparaciones los decisores pueden usar datos concretos de los elementos o realizar juicios de importancia, pero es importante que el valor final de lo que vale un elemento respecto a otro esté totalmente consensuado. Es esencial para el AHP que los juicios humanos, y no sólo la información subyacente, puedan ser usados para realizar evaluaciones. Todo esto es reflejado en términos cuantitativos mediante la asignación de prioridades a los nodos. Las prioridades son números asignados a los nodos que representan el valor de los pesos relativos de los nodos en cualquier grupo. Por definición, la prioridad del objetivo, el nodo padre, es 1.000. Así que las prioridades del hijo deben sumar siempre 1.000 aunque no tiene que ser equitativo el peso de cada una sino que puede haber una que tenga más que otra y viceversa.

\section{IMPLEMENTACIÓN DEL CUADRO DE MANDO}

\subsection{DJANGO}

Django es un framework para hacer aplicaciones webs escrito en Python, gratuito y opensource, basado en el patrón de diseño Modelo-vistacontrolador (mvc). Este es un patrón de arquitectura software que separa los conceptos de la lógica de la aplicación de la lógica de la vista de la aplicación. El funcionamiento de cada una de las partes es sencillo. Cuando el usuario envía una petición por navegador el controlador es quien se encarga de responder a la solicitud, porque es quién controla la lógica de la app. Cuando el controlador registra que se le ha hecho una petición le pide al modelo la información necesaria. El modelo se encarga obtener los datos de la app, consulta la base de datos y recibe la información requerida, respondiendo al controlador. Una vez el controlador tiene los datos se los manda a la vista, que organiza la información, aplica los estilos y construye la página. La representación visual. Para la vista se usan diferentes lenguajes de programación: HTML, con el que se crea la estructura de la web, CSS, para los estilos y Javascript.

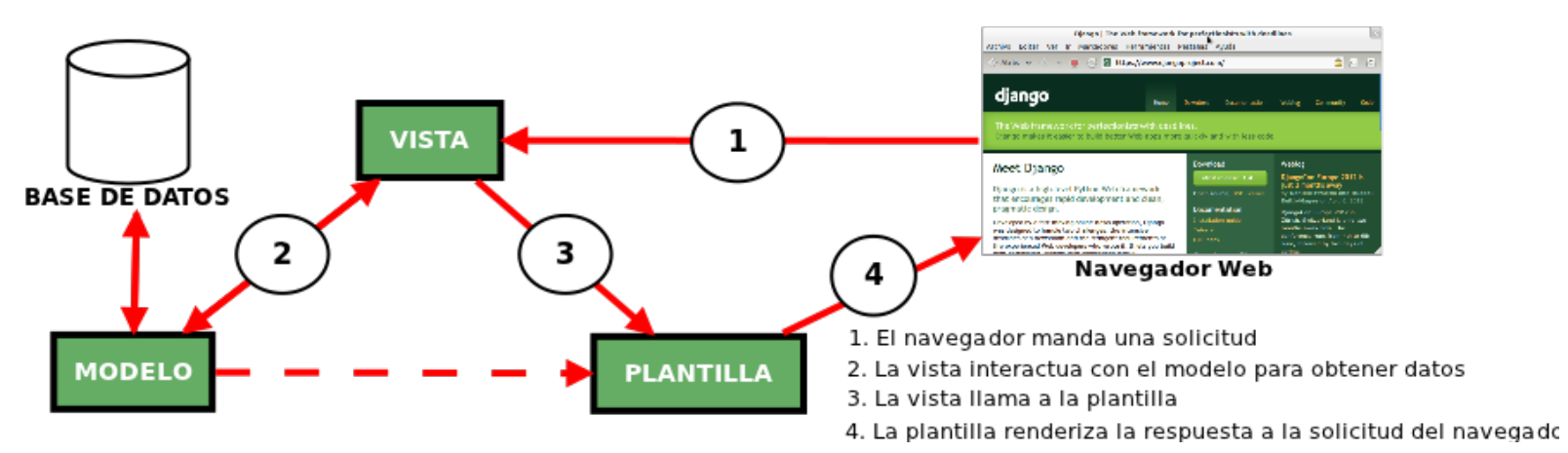

Figura 5: Funcionamiento de un modelo Vista Controlador(MVC).

\subsection{PLOTLY}

Además de los lenguajes convencionales para dar forma a la vista, se utiliza la herramienta Plotly [5] que generar gráficas. El resultado final del trabajo aquí presentado consiste en dotar al experto turístico, además de toda la información existente, de una herramienta atractiva visualmente. Por tanto organizar los resultados en gráficas y analíticas se antoja fundamental.

Plotly puede trabajar con diversos lenguajes, pero en este caso lo integramos con Python. Te permite generar diferentes tipos de gráficas, de barra, de línea, piecharts o mapas. En este caso la cadena que se sigue es sencilla: se piden los datos a la base de datos, en este caso Elasticsearch, y se descargan los datos, en este caso en formato JSON [4]. Aunque Plotly es capaz de integrar datos de otro tipo, como excels o ficheros de texto.

Para generar las diferentes gráficas Plotly tira de ReactJS. ReactJS es una librería de javascript enfocada a la visualización, únicamente. Por tanto a diferencia otros conocidos Frameworks de JS, como AngularJS, no es un modelo-vista-controlador, 
sólo la vista. El secreto de esta librería se basa en cómo gestiona el DOM, el cuerpo del html. Las herramientas convencionales basadas en js hacen los cambios de forma dinámica y en vivo, renderizando el DOM a cada cambio, y sin embargo react hace una copia en memoria del original para que la comparación de cambios sea más rápida otorgando mayor fluidez, siendo más eficiente en la práctica final.

Plotly aporta una serie de funciones con los que poder indicar los componentes que van a aparecer en cada una de las gráficas y su estilo. Aunque también te permite, en el caso de que te parezca insuficiente, crear tus propios componentes en len- guaje reactJS, aportando flexibilidad y ampliando las posibilidades de las gráficas y estilos que te vienen hechas. Además, integra en ocasiones herramientas terceras en su sistema que mejoran el resultado. Como es el caso de Mapbox la hora de generar mapas dinámicos.

Mapbox es un opensource que provee de mapas on-lines. Te otorga un catálogo de mapas con diferentes estilos, además de permitirte poder modificar los estilos del mapa según te parezca de forma manual. Esto lo hace perfecto para poder obtener un producto totalmente personalizado y de una manera relativamente sencilla. Además de ser gratuito.

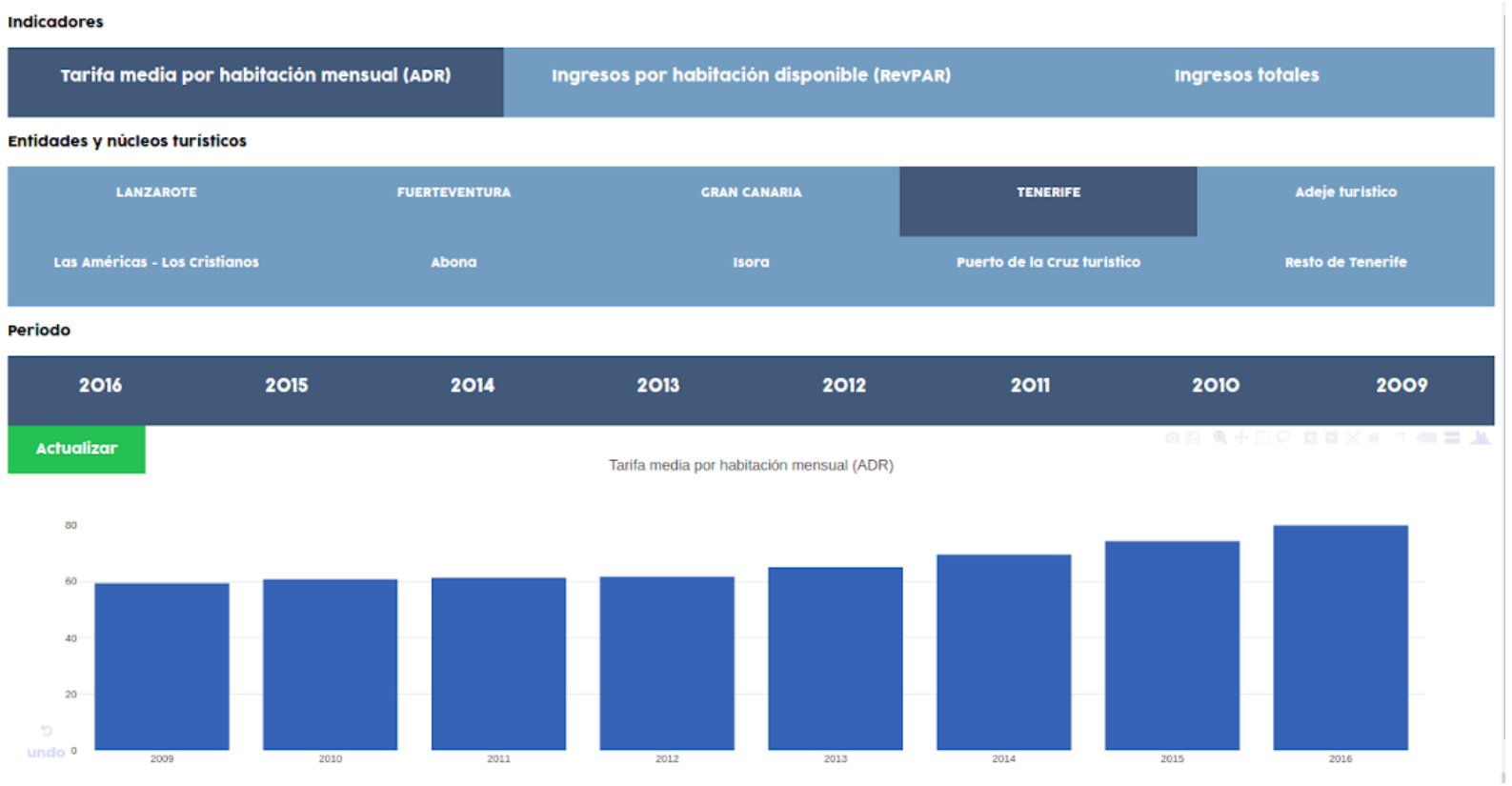

Figura 6: Gráfica dinámica generada a partir de Plotly. Corresponde al KPI Ingresos, de Hoteles, agrupada dentro de Comportamiento Turístico.

\section{CONCLUSIONES}

En el mundo en el que vivimos hay cada vez más fuentes y cantidad de información. Localizar todas las posibles fuentes de información es una ardua tarea, y tomar una decisión sobre las estrategias a seguir también. Estos grandes inconvenientes requieren de la integración de la nuevas tecnologías y de abandonar los métodos tradicionales y arcaicos. Sin embargo, en el mundo del turismo no hay herramientas que hagan esta tarea eficientemente en nuestro país. Las hay que lo hacen parcialmente, aglutinando datos provenientes de fuentes oficiales estructuradas. Sin embargo no existe ninguna que incluya también los datos no estructurados provenientes, por ejemplo, de redes sociales. En este trabajo, proponemos una solución eficaz que ayudará a en última instancia a expertos del sector a tomar decisiones de forma más eficaz. Mediante un cuadro de mando personalizado al sector turístico de la isla de Tenerife.

Se define para ello los KPIs más importantes, consensuados con expertos del sector, para a partir de los cuales buscar las fuentes de información oportunas a cada KPI y poder realizar un seguimiento de éstas. Una búsqueda constante de nuevos datos que se realiza de forma automática gracias a la herramienta Airflow, que ejecuta tareas periódicamente en un orden específico. Datos que se almacenan en el servidor Elasticsearch, que tiene capacidad de multitenencia y en formato JSON. Para después, finalmente, exponerlos en una aplicación web, trabajada con un modelo-vista-controlador que en este caso será Django. Escrito en Python, nos permite generar la infraestructura necesaria 
para poder mostrar los datos expuestos en potentes gráficas visuales creadas mediante la herramienta Plotly basada en reactJS. Además presentamos una clasificación de cada uno de los KPIs mediante la técnica AHP que proporciona una evaluación cuantitativa de los datos mostrados. Esto es mediante comparaciones entre pares de KPIs de forma horizontal, declarando cuantitativamente el orden de importancia de ambos respecto al nodo padre, evitando la intervención de los decisores en esta clasificación. Repitiendo este proceso hasta lograr, en última instancia de estas comparaciones, la puntuación del destino, que nos permite juzgar la salud del mismo.

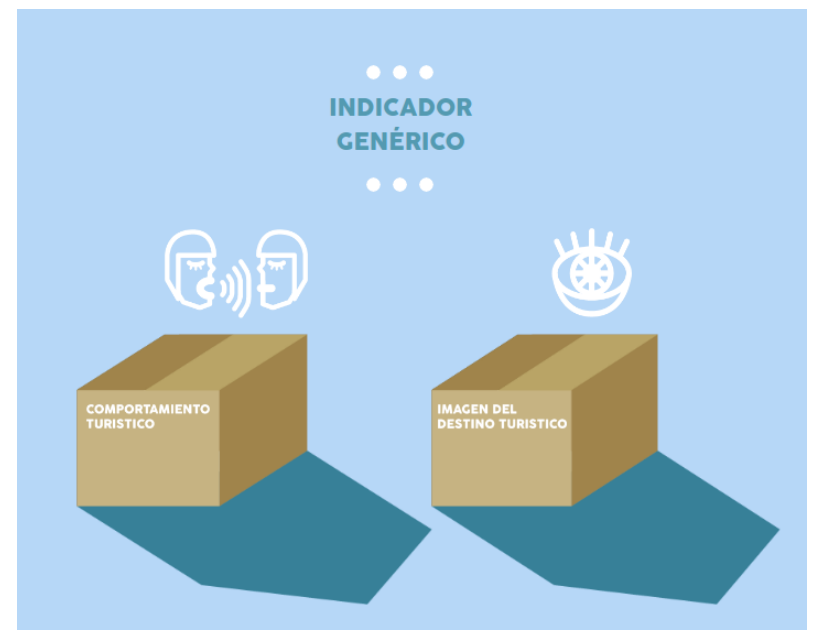

Figura 7: Mediante la técnica AHP se persigue indicar la puntuación del destino para comprobar su estado actual. La puntuación final y de los dos grandes grupos de KPIs va indicada aquí.

\section{Agradecimientos}

Este trabajo ha sido financiado por la Fundación CajaCanarias a través del proyecto titulado "VITUIN: Vigilancia Turística Inteligente de Tenerife en Redes Sociales" n²016TUR15.

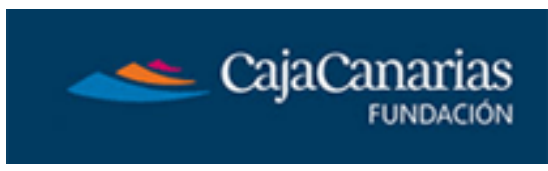

\section{English summary}

\section{DASHBOARD FOR A SMART TOURIST DESTINATIONAbstract}

Improving is always an arduous task that requires new tools and / or techniques that allow the people to give a new approach of the existing problems. Specifically, in activities related to the economic sector, such as the tourism sector, extreme competition demands more than the need to improve every day. It's not a option. It's a obligation. Therefore, predict the direction of the sector and make the right decisions at every moment and juncture is imperatively necessary. For this, the information that the decision makers have is fundamental. We are currently experiencing an explosion in the number of sources of information. But this, in addition to an advantage, brings with it a problem: they are diversified in different places and formats. This paper presents a dashboard that allows the visualization of data collected automatically, from both structured and unstructured sources of the tourist sector in Tenerife, analyzed and classified. All conformed by free software tools.

Keywords: dashboard, analytic hierarchy process, machine learning, key performance indicator, social networks, big data, tourism

\section{Referencias}

[1] Ayuntamientos de Badajoz y Elva, (2017) Sistema de inteligencia turística BadajozElva, http://www.sibadajozelvas.es/.

[2] Cabildo de Fuerteventura, (2017) Portal de inteligencia turística Badajoz, http://www.fuerteventuraintel.es/.

[3] J.R.S.C., M., (2012) Multi Criteria Analysis in the Renewable Energy Industry, Springer, London.

[4] Marrs, T., (2017) JSON at Work, O'Reilly Media

[5] Plotly (2018) Modern visualiazation for the data era, https://plot.ly/ 